\title{
Predominant Enhancement of Glucose Uptake in Astrocytes versus Neurons during Activation of the Somatosensory Cortex
}

\author{
Julien Chuquet, Pascale Quilichini, Esther A. Nimchinsky, and György Buzsáki \\ Center for Molecular and Behavioral Neuroscience, Rutgers University, Newark, New Jersey 07102
}

\begin{abstract}
Glucose is the primary energetic substrate of the brain, and measurements of its metabolism are the basis of major functional cerebral imaging methods. Contrary to the general view that neurons are fueled solely by glucose in proportion to their energetic needs, recent in vitro and ex vivo analyses suggest that glucose preferentially feeds astrocytes. However, the cellular fate of glucose in the intact brain has not yet been directly observed. We have used a real-time method for measuring glucose uptake in astrocytes and neurons in vivo in male rats by imaging the trafficking of the nonmetabolizable glucose analog 6-deoxy- $N$-(7-nitrobenz-2-oxa-1,3-diazol-4-yl)-aminoglucose (6-NBDG) using two-photon microscopy. During resting conditions we found that astrocytes and neurons both take up 6-NBDG at the same rate in the barrel cortex of the rat. However, during intense neuronal activity triggered by whisker stimulation, astrocytes rapidly accelerated their uptake, whereas neuronal uptake remained almost unchanged. After the stimulation period, astrocytes returned to their preactivation rates of uptake paralleling the neuronal rate of uptake. These observations suggest that glucose is taken up primarily by astrocytes, supporting the view that functional imaging experiments based on glucose analogs extraction may predominantly reflect the metabolic activity of the astrocytic network.
\end{abstract}

\section{Introduction}

Glucose is the main source of energy for the brain (Sokoloff, 1977). The spatiotemporal coincidence of glucose utilization with localized neuronal activation, or "neuro-metabolic coupling" underlies the interpretation of widely used functional imaging methods of the human brain (Raichle and Mintun, 2006). Thus, a significant portion of our knowledge of intact brain function depends on an understanding of localized glucose processing by brain cells. Because clinically used imaging tools do not possess cellular-scale resolution, studying the fate of glucose in the brain parenchyma after it has left the vascular compartment represents a challenging task (Nehlig and Coles, 2007). The traditional method for measuring glucose metabolism is the autoradiography of brain slices obtained from animals perfused in vivo by systemic injection of 2-deoxyglucose (2-DG) (Sokoloff, 1977), which at best provides submillimeter resolution. The observation that 2-DG labeling in the cortex is less intensely present in neuronal somata than in the neuropil, which is rich in synapses and astrocytes, has led to the proposition that astrocytes, despite their apparent passivity, may be important users of glucose (McCasland, 1996). Astrocytes' avidity for glucose has been observed in tissue culture where the basal rate of glucose utilization is 3-10 times higher than in neurons (Lopes-Cardozo

Received Feb. 11, 2010; revised May 28, 2010; accepted July 29, 2010.

J.C. is supported in part by the Philippe Foundation. We thank Patrick Hof, Omar Touzani, Luc Pellerin, and Nicolas Liaudet for constructive comments and thoughtful discussions.

Correspondence should be addressed to Dr. Julien Chuquet, Center for Molecular and Behavioral Neuroscience, Rutgers University, 197 University Avenue, Newark, NJ 07102. E-mail: julienchuquet@hotmail.com.

DOI:10.1523/JNEUROSCI.0762-10.2010

Copyright $\odot 2010$ the authors $\quad 0270-6474 / 10 / 3015298-06 \$ 15.00 / 0$ et al., 1986; Magistretti and Pellerin, 1996a). These ex vivo or in vitro findings converge on the view that neurons, despite their higher energetic needs (Attwell and Laughlin, 2001), may have a much lower glucose utilization than astrocytes (Herrero-Mendez et al., 2009). However, this is contradicted by other studies showing that neurons are the primary site of glucose uptake and utilization (Brookes and Yarowsky, 1985; Itoh et al., 2004). Yet, direct in vivo evidence of such a compartmentalization of glucose utilization, if any, is still lacking (Hyder et al., 2006; Nehlig and Coles, 2007). 2-DG analyses, using higher spatial-resolution methods combined with immunohistochemical labeling, indicate that neurons and glia may have at rest an equal share of glucose utilization (Nehlig et al., 2004).

To address the role of glucose in fueling neuronal activity, more direct methods in the living brain are needed (Nehlig and Coles, 2007). We hypothesized that the nonmetabolizable fluorescent glucose analog 6-deoxy- $N$-(7-nitrobenz-2-oxa-1,3-diazol-4-yl)aminoglucose (6-NBDG) (Speizer et al., 1985), combined with high spatial-resolution, real-time two-photon microscopy, would be effective in addressing this issue in the living brain. Fluorescent glucose analogs have been increasingly used for studying glucose transport and metabolism in many cell types besides astrocytes and neurons (Shimada et al., 1994; Porras et al., 2004; Rouach et al., 2008). Here, we examine the kinetics of 6-NBDG uptake into astrocytes and neurons in the rat barrel cortex, during both resting conditions and sensory stimulation.

\section{Materials and Methods}

Surgical procedure. All experimental procedures were performed according to the animal use and welfare guidelines of Rutgers University. Male 


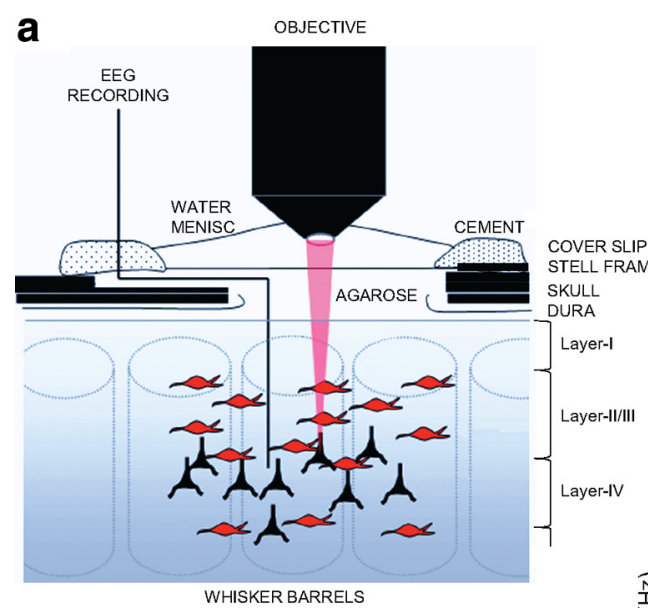

b
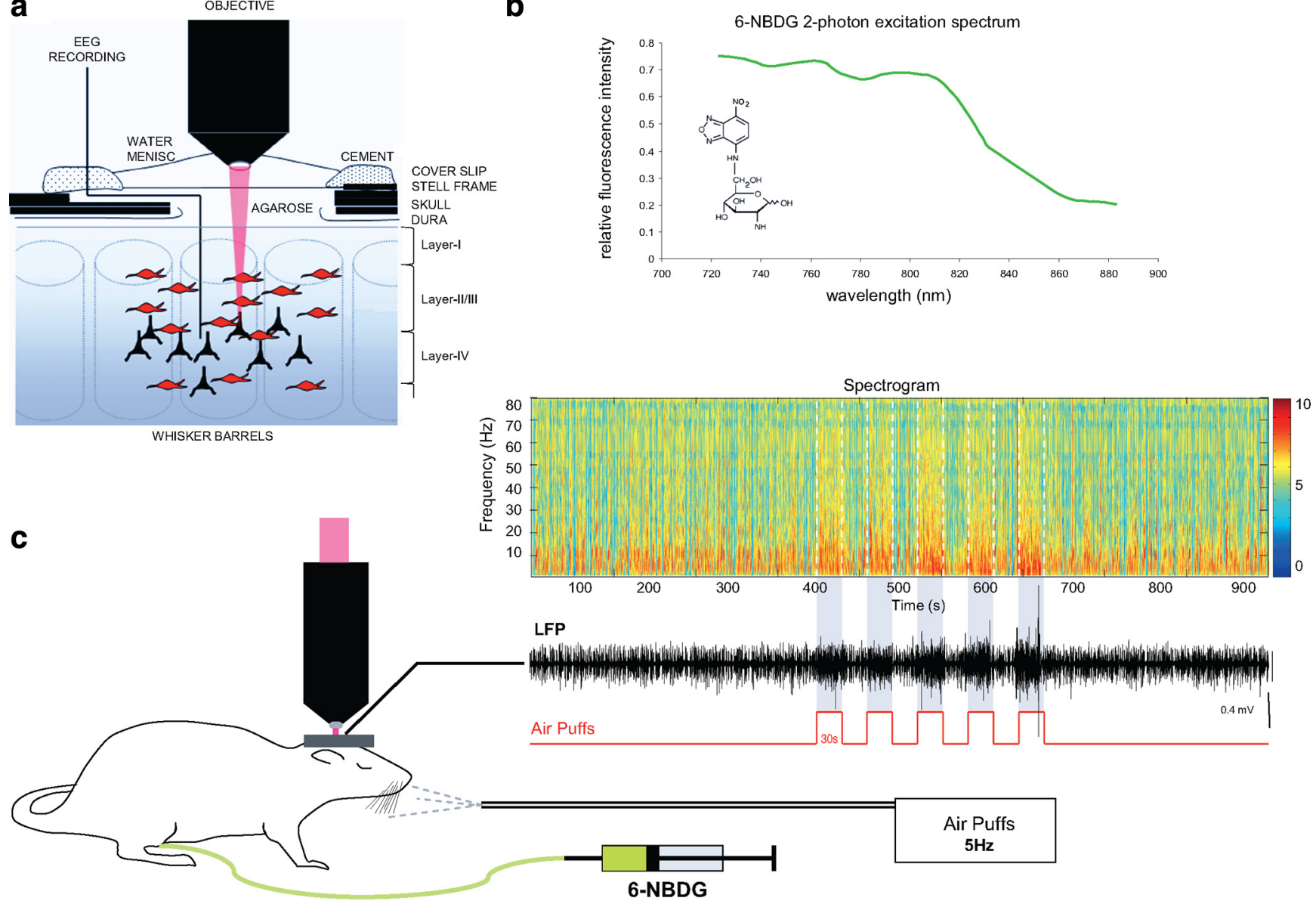

Figure 1. 6-NBDG two-photon microscopy experimental set-up. $\boldsymbol{a}$, Detail of the cranial window preparation mounted over the barrel cortex. A tungsten wire ( $50 \mu \mathrm{m}$ diameter) was inserted close to the imaging area to record the LFP in layer II-III of the barrel cortex. Agarose filled the chamber to stabilize the brain from cardiorespiratory movements. Astrocytes were labeled using SR-101. $\boldsymbol{b}$, Characterization of 6-NBDG two-photon excitation in vivo. $\boldsymbol{c}$, Experimental arrangement for simultaneous LFP recording and two-photon imaging during whisker stimulation. 6-NBDG was infused to the femoral vein. The whiskers of the contralateral side were mechanically stimulated by air puffs generating sustained network activity in the LFP. The time-frequency spectrogram of the LFP showed an increase in the LFP power in the $7-20 \mathrm{~Hz}$ range during each period of $30 \mathrm{~s}$ of whisker stimulation.

Sprague Dawley rats (50-100 g) were anesthetized with urethane (1.7 $\mathrm{g} / \mathrm{kg}$, i.p.). Animals were tracheotomized and mechanically ventilated. The pulmonary volume was set, and blood gas components were monitored $\left(\mathrm{PaCO}_{2}\right.$ between 35 and $45 \mathrm{mmHg}, \mathrm{PaO}_{2}$ between 100 and 200 $\mathrm{mmHg}, \mathrm{pH}$ between 7.35 and 7.50). The femoral artery was cannulated to permit vascular access and the monitoring of the mean arterial pressure (maintained between 75 and $100 \mathrm{mmHg}$ ). The rectal temperature was monitored and kept close to $37.5^{\circ} \mathrm{C}$ with a heating pad (Harvard Apparatus). A steel frame $(1.5 \times 6 \mathrm{~cm})$ with a hole $(4 \mathrm{~mm}$ diameter $)$ centered over the right barrel cortex $(-2.5 \mathrm{~mm}$ posterior to the bregma and $4.9 \mathrm{~mm}$ lateral from midline) was attached on the skull with dental cement. A craniotomy was performed, and the dura mater was removed. After the labeling procedure for astrocytes, the craniotomy was filled with agarose (Sigma; type III-A, $0.75 \%$ in a HEPES-buffered solution) and covered with a coverslip immobilized with dental cement.

Labeling of astrocytes and 6-NBDG administration. Astrocytes were labeled by bathing the cortical surface with SR-101 $(1 \mu \mathrm{M})$ in HEPES buffer, pH 7.42 (Sigma), for $10 \mathrm{~min}$ (Nimmerjahn et al., 2004). 6-NBDG (Invitrogen) was dissolved in a solution of $55 \%$ of HEPES buffer and $45 \%$ of DMSO, pH 7.42, to a concentration of $5 \mathrm{mg} / \mathrm{ml}$. The femoral vein was cannulated, and 6-NBDG was injected with an infusion pump at a rate of $100 \mu \mathrm{l} / \mathrm{min} / 100 \mathrm{~g}$ for bolus experiments or $20 \mu \mathrm{l} / \mathrm{min} / 100 \mathrm{~g}$ for continuous infusion experiments.

Whisker stimulation. The whiskers on the left of the animal's snout were stimulated with puffs of air produced by a picospritzer (Harvard Apparatus) coupled to a stimulator (Master 8; DL Instrument International). Air was ejected at 1 bar via plastic tubing ( $1.5 \mathrm{~mm}$ diameter) positioned $\sim 1 \mathrm{~cm}$ lateral and anterior to the animal's nose to stimulate the whole whisker pad. Whiskers were stimulated at $5 \mathrm{~Hz}$ for $30 \mathrm{~s}$ (pulse width $=100 \mathrm{~ms}$ ) repeated five times with periods of $30 \mathrm{~s}$ of rest between each block of stimulation. Online display of the local field potential (LFP) was used to monitor the presence of increased spindle-like activity during the whisker stimulations.

EEG recording. A tungsten wire ( $50 \mu \mathrm{m}$ in diameter) was inserted close $(\leq 500 \mu \mathrm{m})$ to the imaging area and sealed underneath the coverslip window to record the LFPs in layer II-III of the barrel cortex. The neurophysiological signal was acquired at $2 \mathrm{kHz}$ with a DAM- $80 \mathrm{AC}$ amplifier (WPI) and Data-Trax acquisition software (WPI). The wide-band signal was downsampled to $1250 \mathrm{~Hz}$ and used as LFP signal. Data analysis was performed with custom-written Matlab software (The Mathworks).

The LFP displayed a slow oscillation activity during anesthesia, and the amount of urethane was adjusted for keeping the plane of anesthesia at constant level. Spindle-like activity was observed spontaneously and was associated with a deflection of the contralateral whiskers to craniotomy but not in response to the stimulation of the ipsilateral whiskers (Fig. 1c).

Two-photon laser scanning microscopy. Imaging was performed with a custom-built two-photon laser (Chameleon; Coherent) scanning microscope consisting of an Olympus trinocular, Cambridge scan mirrors, and a XLUMPFLN-20XW Olympus objective. Fluorescence emission was directed by a 700-LP dichroic, split with a 565-LP dichroic into green and red channels, and further restricted with BGG22 and HQ607/45 filters placed before the green and red photomultipliers (Hamamatsu), respectively. Frames $(512 \times 512$ pixels $)$ were collected at a frequency of 0.016 $\mathrm{Hz}$ (bolus experiment) or $0.32 \mathrm{~Hz}$ (activation experiment). 
a
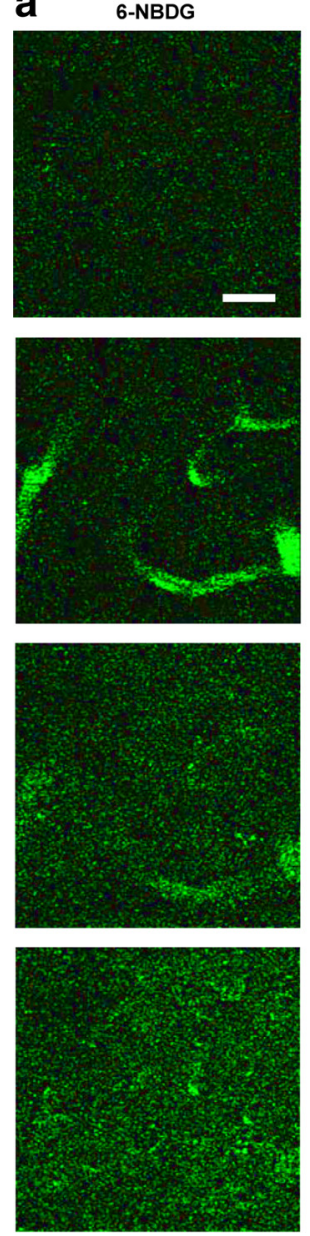

SR-101
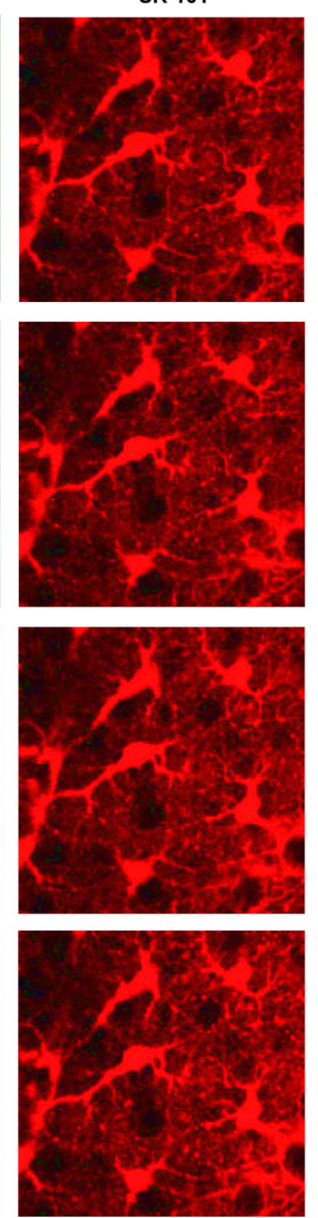

merge
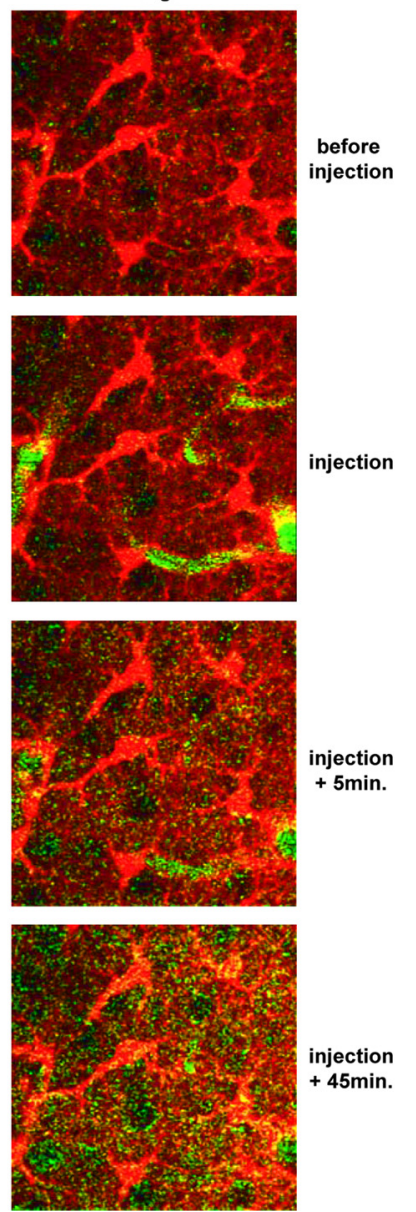

injection

injection
$+5 \mathrm{~min}$. b
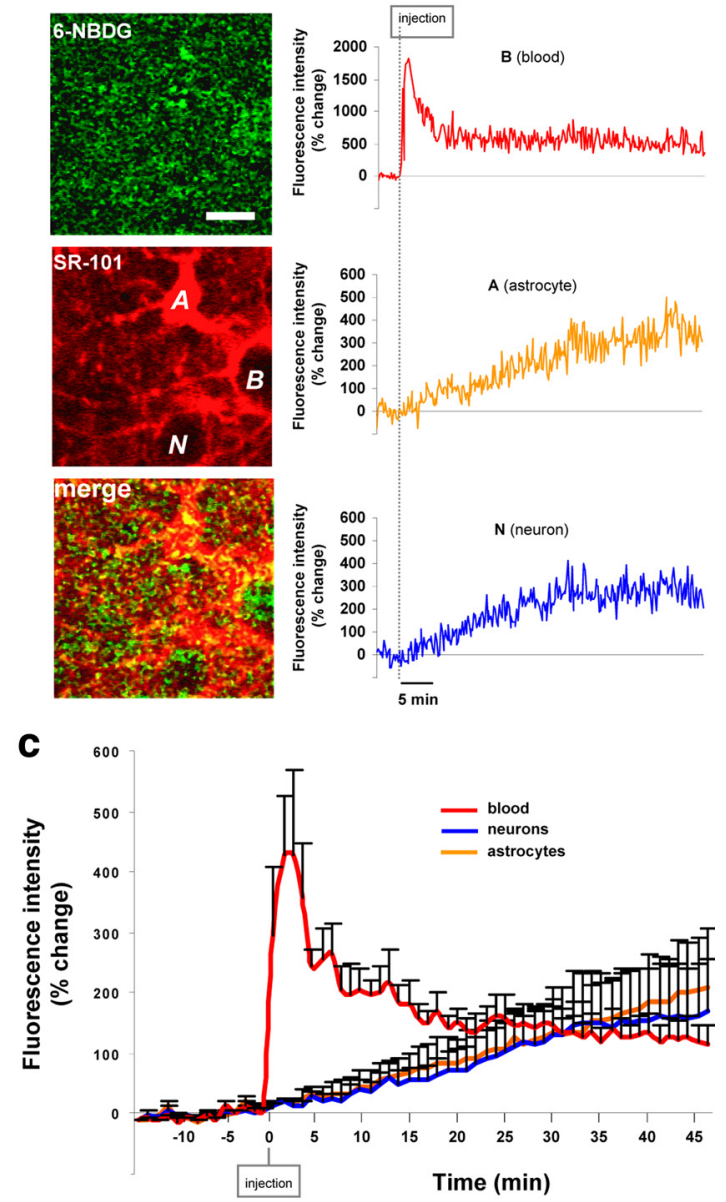

Figure 2. Uptake of 6-NBDG in resting condition. $\boldsymbol{a}$, Time-lapse sequence of 6-NBDG invading the brain parenchyma (see also supplemental video available at www.jneurosci.org as supplemental material). Green channel shows the blood vessel shape is revealed by 6-NBDG fluorescence at the time of injection. Red channel shows contrast with SR-101 astrocytic staining; neuronal somata appear as dark gaps. Scale bar, $20 \mu \mathrm{m} . \boldsymbol{b}$, Kinetic of 6-NBDG uptake in the vascular compartment (B), in an astrocyte (A), and in a neuron (N). Scale bar, $10 \mu \mathrm{m}$. $\boldsymbol{c}$, Astrocytes (in orange) and neurons (in blue) ( 31 and 30 cells from six animals, respectively) showed similar kinetics of uptake over $45 \mathrm{~min}$.

Data analysis. Neurons and astrocytes were distinguished by using the red signal emitted by SR-101. Astrocytes concentrate SR-101, and their somata appear intensely bright. In contrast, neurons exclude the dye and appear as black holes (Nimmerjahn et al., 2004). Each image of the sequence was aligned over the previous image with Align Slice (ImageJ, National Institutes of Health) to correct $x-y$ deviation caused by possible drift of the tissue. Fluorescence intensity was measured in a region of interest (average intensity over $\sim 250$ pixels) strictly limited to the somatic area. Signals were expressed as relative fluorescence changes $(\Delta F /$ $F_{0}$ ), where $F_{0}$ was the mean of the baseline period. Slope was determined after the equation of the linear regression line through the data points.

Statistics. The results are presented as mean \pm SEM. Statistical analyses were performed with an unpaired Student's $t$ test after the demonstration of homogeneity of variance with a one-way ANOVA or a repeatedmeasure ANOVA. The Bonferroni test was used for post hoc testing. The significance level was set at $p=0.05$ (two-tailed).

\section{Results}

Uptake of 6-NBDG in resting condition

$6-N B D G$ in solution was best excited at wavelengths $<820 \mathrm{~nm}$ (Fig. $1 b$ ). We set the excitation wavelength at $810 \mathrm{~nm}$ to stray from the autofluorescence zone around $830 \mathrm{~nm}$. The barrel somatosensory cortex was imaged at a depth of $250-350 \mu \mathrm{m}$ on a field containing several astrocytes and neurons $(2.1 \pm 0.6$ and $2.1 \pm 0.5$ cells per field of imaging, respectively) and at least one blood vessel to control for the 6-NBDG arrival into the local microcirculation (Fig. $2 a$ ). A bolus of $2 \mathrm{mg} / 100 \mathrm{~g}$ of $6-\mathrm{NBDG}$ was injected intravenously at a rate of $100 \mu \mathrm{l} / \mathrm{min} / 100 \mathrm{~g}$. Considering that the blood volume (in milliliters) is $7-8 \%$ of body weight and assuming a normal glycemia $(0.38-0.55 \mathrm{mmol} / 100 \mathrm{ml}$ of blood), the equivalent increase of blood glucose at the end of the injection of $6-\mathrm{NBDG}$ was $0.07 \mathrm{mmol} / 100 \mathrm{ml}$. Once the bolus started, fluorescence could readily be seen filling up the local vascular tree. For the measurement of 6-NBDG fluorescence intensity, a square region of interest was chosen inside one or more vessels; for both cell types this square was limited strictly to the somatic area. As NBDG fluorescence progressively invaded the brain parenchyma, the whole field of observation appeared in a homogeneous manner without a visually obvious "hot spot" (supplemental video, available at www.jneurosci.org as supplemental material, and Fig. 2a). Astrocytes and neurons (six animals, 13 astrocytes, and 13 neurons) showed similar kinetics of uptake over the 45 min observation period (Fig. 2c).

\section{Uptake of 6-NBDG during whisker stimulation}

The aim of the second set of experiments was to analyze the kinetic of 6-NBDG uptake in astrocytes and neurons during physiological activation (Fig. 1c). After a stabilization period of $15 \mathrm{~min}, 6-\mathrm{NBDG}(5 \mathrm{mg} / \mathrm{ml})$ was injected into the femoral vein at the constant flow of $20 \mu \mathrm{l} / \mathrm{min} / 100 \mathrm{~g}$ of body weight until the end 

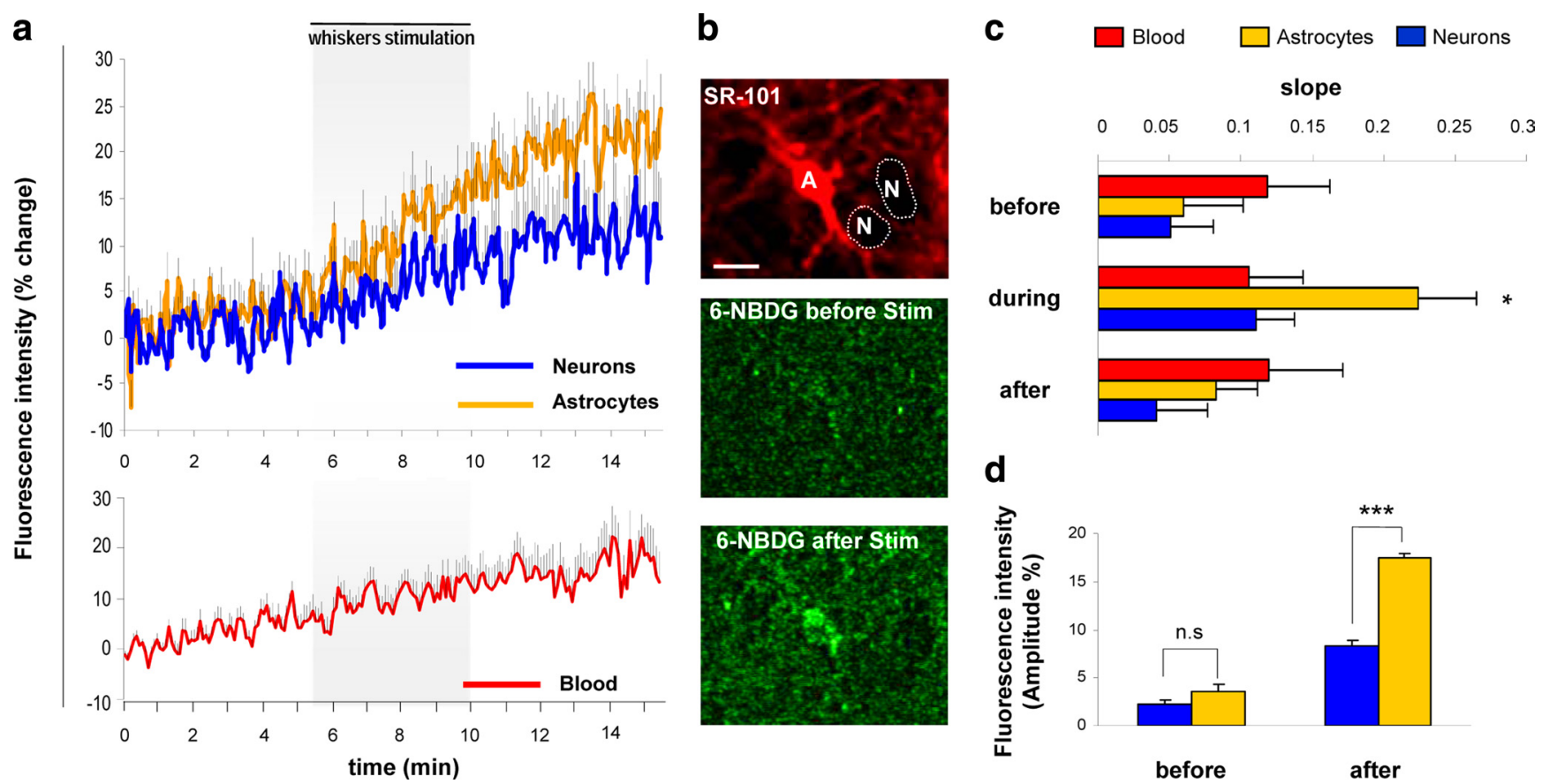

Figure 3. Uptake of 6-NBDG during whisker stimulation (WS). $\boldsymbol{a}$, Fluorescence intensity changes of 6-NBDG in astrocytes, neurons, and blood (red trace, bottom) during functional activation of the barrel cortex. $\boldsymbol{b}$, Preferential accumulation of 6-NBDG in astrocytes after functional activation. Top, SR-101 astrocytic staining (A for astrocyte and N for neurons); neuronal somata appear as dark gaps. Middle and bottom, Average fluorescence of 6-NBDG over 75 s before and after the stimulation period. Scale bar, $10 \mu$ m. $c$, Slope measurement of 6-NBDG uptake before, during, and after WS. Before WS, both cell types showed a similar slope of 6-NBDG uptake. During WS, only the astrocytic 6-NBDG uptake slope significantly increased ( $p<0.05$ ). After WS, the slope uptake returned to a prestimulation level for both cell types. $\boldsymbol{d}$, Comparison of fluorescence level in cells (astrocytes in orange and neuron in blue) showed that astrocytes take up significantly more 6-NBDG than do neurons $(p<0.001)$ during functional activation.

of experiment to create a steady delivery of 6-NBDG to the brain (Fig. 3a). As in the bolus experiment described above, the two cell types showed similar uptake rate with no significant difference between slopes $(0.06 \pm 0.04$ for astrocytes and $0.05 \pm 0.03$ for neurons; $p>0.05$ ) (Fig. $3 a, c$ ) and between fluorescence amplitude $(3.57 \pm 0.68 \%$ for astrocytes and $2.15 \pm 0.57 \%$ for neurons; $p>0.05$ ) (Fig. $3 d$ ) in the baseline condition. Whisker stimulation started 10 min after the beginning of 6-NBDG infusion, whereas the loading rate in the parenchyma showed a steady slope (slope changes not significantly different from 0 after 6 min of infusion; $n=4$ animals). Air puffs triggered a sustained evoked responses in the LFP recorded in layer II-III of the barrel cortex, also reflected by an increased LFP power in the $7-20 \mathrm{~Hz}$ range (Fig. 1c). Such an increase of network activity was strictly observed during stimulation of the contralateral whisker pad relative to the recording site, but not during the stimulation of the ipsilateral whisker pad. Arterial pressure did not show consistent changes during the activation. During the stimulation of the whisker pad, 6-NBDG uptake slope significantly increased in astrocytes (from $0.06 \pm 0.04$ to $0.22 \pm 0.04 ; p<0.001$ ) but nonsignificantly in neurons (from $0.05 \pm 0.03$ to $0.11 \pm 0.03 ; p>0.05$; six animals, 31 astrocytes, and 31 neurons). After the stimulation, both slopes decreased to their prestimulation level. There was no difference between the fluorescence intensity level of astrocytes and neurons just before the stimulation (mean $>75 \mathrm{~s}$ ) (see Fig. $3 d$ ). However, just after the stimulation, astrocytes showed a much greater accumulation of 6-NBDG than neurons (mean $>75$ s; $p<0.001$ ) (Fig. 3b).

\section{Discussion}

We present a novel approach for studying brain glucose uptake in vivo with high spatial and temporal resolution. We show that the glucose analog 6-NBDG is detectable in the brain at a subcellular scale, seconds after its intravenous administration at physiologically relevant concentrations. Our study is the first real-time imaging of the cellular supply of a glucose analog in the intact brain. We found that 6-NBDG homogeneously invades the parenchymal compartment and reaches astrocytes and neurons at the same rate in the absence of triggered neuronal work. This is in agreement with previous ex vivo observations showing that 45 min after its systemic injection the radioactive glucose analog $2-D G$ is detected equally in astrocytes and neurons (Nehlig et al., 2004). 6-NBDG is a nonmetabolizable analog of glucose whose uptake is affected only by glucose transporter activity. Fluorescent glucose analogs enter cells through glucose transporters with an affinity 300 times higher than D-glucose, albeit at a much slower rate (Yamada et al., 2000; Barros et al., 2009). It has been demonstrated in vitro that glucose inhibits 6-NBDG uptake with the same efficiency in astrocytes and neurons (Aller et al., 1997), suggesting that 6-NBDG would compete with glucose equally for transport into the two cell types. However, the concentration of 6-NBDG used here represents only $5 \%$ of the glycemia. It is therefore unlikely that $6-\mathrm{NBDG}$ would compete with blood-borne glucose and would modify the kinetic of its transport and metabolism.

The barrel cortex is among the best characterized sensory areas of the rat brain. Activation of this cortical area generates a well understood neuronal network activity (Contreras and Steriade, 1996). Because this system produces attenuation of neuronal responses to sustained whisker deflection (Chung et al., 2002) we avoided habituation by stimulating the whiskers intermittently at a low frequency. This approach produced a steady level of activation as evidenced by spectral analysis of LFP and increased firing 
frequency of layer II-III and IV neurons. In contrast to the resting conditions where 6-NBDG uptake rate was similar in astrocytes and neurons, we found that during whisker stimulation-evoked neuronal activity the supplementary extraction of 6-NBDG essentially took place in astrocytes, whereas the neuronal extraction rate displayed only a tendency to increase. This activationmediated differential enhancement of glucose uptake in astrocytes and neurons has been reported previously in several in vitro studies. As pioneers in the use of fluorescent glucose to investigate brain glucose metabolism, Barros and coworkers have shown in vitro that glutamate-induced neuronal firing surprisingly mediates acute inhibition of glucose transport in neurons while stimulating astrocytic glucose uptake (Loaiza et al., 2003; Porras et al., 2004). Thus, it seems likely that during neuronal activation there is a redistribution of glucose away from neurons and toward astrocytes. In line with this, a novel computation of experimental datasets from rodents and humans predicted that when the activity level of the brain increases the proportion of glucose entering into glia also increases (Jolivet et al., 2009). In addition, further work suggests that in acute cerebellar slices Bergmann glial cells transport and metabolize fluorescent hexoses faster than both Purkinje and granule cells (Barros et al., 2009). Therefore, during activation, in addition to an increase in the delivery of glucose caused by local hyperemia, those results are suggestive of an active regulation of the transport of glucose into astrocytes and neurons.

We have to point out two potential caveats in the interpretation of our results. First, it is possible that anesthesia modifies the rules of glucose extraction and trafficking. Future studies should be conducted either with a different class of anesthetic agents or, preferably, without anesthesia to confirm the present findings (Oz et al., 2004). Second, most energy metabolism may take place at the level of synapses rather than at or around the cell bodies of astrocytes and neurons, where our measurements were taken. However, given its high diffusion rate, 6-NBDG is expected to distribute homogeneously in all compartments of the cell (Barros et al., 2009).

Whatever the mechanism of glucose distribution between astrocytes and neurons, our data raise the question of whether brain cells take up glucose in proportion to their energetic needs. The energetic needs of astrocytes and neurons is still an open debate because calculations critically depend on various assumptions and experimental conditions. At rest we found fluorescence intensity of 6-NBDG to be similar in astrocytes and neurons, suggesting the need for glucose is the same in both cell types. In rats, the volume occupied by astrocytes in vivo is estimated to be $\sim 30 \%$ of the cortical gray matter cellular volume (not counting extracellular space) (Norenberg, 1994; Hertz, 2008). According to the measurement of acetate oxidation, which is selectively oxidized by astrocytes, oxidative metabolism of astrocytes also accounts for $\sim 30 \%$ of total brain cell oxidative metabolism in rodents (Sibson et al., 2001; Oz et al., 2004; Cruz et al., 2005; Patel et al., 2005). Therefore, the level of oxidative metabolism in astrocytes and neurons may not differ greatly in resting conditions consistent with similar 6-NBDG uptake found here in both cell types. During stimulation, the paralleled uptake of glucose analog by astrocytes and neurons is no longer observed: a vast majority of the additional glucose is taken up by astrocytes. A first possible interpretation is that astrocytes take up more glucose during functional activation because they spend more energy than active neurons. In support of this, it has been shown that the oxidative metabolism of astrocytes increases during brain activation, suggesting the astrocytic tricarboxylic acid cycle is not saturated in resting condition and may support the extra work resulting from neuronal activation (Cruz et al., 2005; Wyss et al., 2009). Interestingly, it has been proposed that the multitude of astrocytic tasks unraveled such as active clearance of $\mathrm{K}^{+}$(Somjen et al., 2008) or fast and local calcium signaling (Chuquet et al., 2010) could altogether account for a high energetic expense and therefore a high need of glucose by astrocytes (Hertz et al., 2007). However, although astrocytic energy needs could have been underestimated, it is unlikely that their energy expenditure overcomes the neuronal one. A general consensus is that astrocytes contribute significantly less to ATP production than neurons during spiking activity (Attwell and Laughlin, 2001; Hyder et al., 2006; Jolivet et al., 2009). Therefore, our demonstration that glucose is preferentially taken up by astrocytes during enhanced neuronal activity represents a paradox. Considering that glucose is the almost exclusive energy substrate used by the brain, one explanation to resolve this paradox lies in the proposal that lactate, derived from the glucose extracted by astrocytes, may be synthesized and delivered to neurons to complement their energy budget (Magistretti and Pellerin, 1999; Pellerin et al., 2007). The corollary of this is that neurons may use less glucose than predicted by their energy requirement in agreement with the present finding. Indeed, neurons have a lower glycolytic rate than astrocytes (Almeida et al., 2001), which has been explained by the finding that the enzyme 6-phosphofructo-2-kinase/fructose-2,6biphosphatase-3, a key activator of glycolysis, is active only in astrocytes (Herrero-Mendez et al., 2009). The present data are compatible with the astrocyte-to-neuron shuttle hypothesis originally conceptualized by Magistretti and Pellerin (1999) (Hyder et al., 2006) and provide the first in vivo evidence of a preferential transport of glucose to astrocytes when neuronal activity is increased. Nevertheless, the contribution of astrocytes to support neuronal energetics is likely to depend on the stimulus paradigm. The flux of lactate from astrocytes to neurons has been suggested to be reversible, depending on the redox state of each cell (NAD+/NADH) (Cerdán et al., 2006; Mangia et al., 2009).

In summary, in vivo 6-NBDG two-photon microscopy represents an effective approach for the study of glucose trafficking in the intact brain. The present study described the uptake kinetics of a glucose analog in astrocytes and neurons in vivo. Under "basal" cortical activity both cell types take up 6-NBDG at the same rate. During intense firing triggered by a sensory stimulation, the uptake is accelerated mainly in astrocytes, supporting the hypothesis that astrocytes play a central role in brain energetics (Voutsinos-Porche et al., 2003). An indirect implication of our findings is that signals detected in functional imaging experiments, using glucose analogs such as in positron emission tomography or 2-DG autoradiography, may predominantly reflect the metabolic activity of the astrocytic network (Magistretti and Pellerin, 1996b).

\section{References}

Aller CB, Ehmann S, Gilman-Sachs A, Snyder AK (1997) Flow cytometric analysis of glucose transport by rat brain cells. Cytometry 27:262-268.

Almeida A, Almeida J, Balaños JP, Moncada S (2001) Different responses of astrocytes and neurons to nitric oxide: the role of glycolytically generated ATP in astrocyte protection. Proc Natl Acad Sci U S A 26:15294-15299.

Attwell D, Laughlin SB (2001) An energy budget for signalling in the grey matter of the brain. J Cereb Blood Flow Metab 21:1133-1145.

Barros LF, Courjaret R, Jakoby P, Loaiza A, Lohr C, Deitmer JW (2009) Preferential transport and metabolism of glucose in Bergmann glia over Purkinje cells: a multiphoton study of cerebellar slices. Glia 57:962-970.

Brookes N, Yarowsky PJ (1985) Determinants of deoxyglucose uptake in cultured astrocytes: the role of the sodium pump. J Neurochem 44:473-479. 
Cerdán S, Rodrigues TB, Sierra A, Benito M, Fonseca LL, Fonseca CP, GarcíaMartín ML (2006) The redox switch/redox coupling hypothesis. Neurochem Int 48:523-530.

Chung S, Li X, Nelson SB (2002) Short-term depression at thalamocortical synapses contributes to rapid adaptation of cortical sensory responses in vivo. Neuron 34:437-446.

Chuquet J, Bhaukaurally K, Santello M, Liaudet N, Bouvier D, Di Castro A, Volterra A (2010) Fast and local calcium transients in astrocyte processes encode synaptic activity in the adult hippocampus. FENS Abstr 5:044.4.

Contreras D, Steriade M (1996) Spindle oscillation in cats: the role of corticothalamic feedback in a thalamically generated rhythm. J Physiol 490: $159-179$.

Cruz NF, Lasater A, Zielke HR, Dienel GA (2005) Activation of astrocytes in brain of conscious rats during acoustic stimulation: acetate utilization in working brain. J Neurochem 92:934-947.

Herrero-Mendez A, Almeida A, Fernández E, Maestre C, Moncada S, Bolaños JP (2009) The bioenergetic and antioxidant status of neurons is controlled by continuous degradation of a key enzyme by APC/C-Cdh1. Nat Cell Biol 11:747-752.

Hertz L (2008) Bioenergetics of cerebral ischemia: a cellular perspective. Neuropharmacology 55:289-309.

Hertz L, Peng L, Dienel GA (2007) Energy metabolism in astrocytes: high rate of oxidative metabolism and spatiotemporal dependence on glycolysis/glycogenolysis. J Cereb Blood Flow Metab 27:219-249.

Hyder F, Patel AB, Gjedde A, Rothman DL, Behar KL, Shulman RG (2006) Neuronal-glial glucose oxidation and glutamatergic-GABAergic function. J Cereb Blood Flow Metab 26:865-877.

Itoh Y, Abe T, Takaoka R, Tanahashi N (2004) Fluorimetric determination of glucose utilization in neurons in vitro and in vivo. J Cereb Blood Flow Metab 24:993-1003.

Jolivet R, Magistretti PJ, Weber B (2009) Deciphering neuron-glia compartmentalization in cortical energy metabolism. Front Neuroenergetics $1: 1-10$.

Loaiza A, Porras OH, Barros LF (2003) Glutamate triggers rapid glucose transport stimulation in astrocytes as evidenced by real-time confocal microscopy. J Neurosci 23:7337-7342.

Lopes-Cardozo M, Larsson OM, Schousboe A (1986) Acetoacetate and glucose as lipid precursors and energy substrates in primary cultures of astrocytes and neurons from mouse cerebral cortex. J Neurochem 46: 773-778.

Magistretti PJ, Pellerin L (1996a) Cellular mechanisms of brain energy metabolism. Relevance to functional brain imaging and to neurodegenerative disorders. Ann N Y Acad Sci 777:380-387.

Magistretti PJ, Pellerin L (1996b) The contribution of astrocytes to the $18 \mathrm{~F}$ 2-deoxyglucose signal in PET activation studies. Mol Psychiatry 1:445452.

Magistretti PJ, Pellerin L (1999) Cellular mechanism of brain energy metabolism and their relevance to functional brain imaging. Philos Trans R Soc Lond B Biol Sci 354:1155-1163.

Mangia S, Simpson IA, Vannucci SJ, Carruthers A (2009) The in vivo neuron-to-astrocyte lactate shuttle in human brain: evidence from modeling of measured lactate levels during visual stimulation. J Neurochem 109:55-62.

McCasland JS (1996) Metabolic activity in antigenically identified neurons: a double labeling method for high-resolution 2-deoxyglucose and immunohistochemistry. J Neurosci Methods 68:113-123.

Nehlig A, Coles JA (2007) Cellular pathways of energy metabolism in the brain: is glucose used by neurons or astrocytes? Glia 55:1238-1250.

Nehlig A, Wittendorp-Rechenmann E, Lam CD (2004) Selective uptake of $\left[{ }^{14} \mathrm{C}\right] 2$-deoxyglucose by neurons and astrocytes: high-resolution microautoradiographic imaging by cellular ${ }^{14} \mathrm{C}$-trajectography combined with immunohistochemistry. J Cereb Blood Flow Metab 24:1004-1014.

Nimmerjahn A, Kirchhoff F, Kerr JN, Helmchen F (2004) Sulforhodamine101 as a specific marker of astroglia in the neocortex in vivo. Nat Methods $1: 31-37$.

Norenberg MD (1994) Astrocyte response to CNS injury. J Neuropathol Exp Neurol 53:213-220.

Oz G, Berkich DA, Henry PG, Xu Y, LaNoue K, Hutson SM, Gruetter R (2004) Neuroglial metabolism in the awake rat brain: CO2 fixation increases with brain activity. J Neurosci 24:11273-11279.

Patel AB, de Graaf RA, Mason GF, Rothman DL, Shulman RG, Behar KL (2005) The contribution of GABA to glutamate/glutamine cycling and energy metabolism in the rat cortex in vivo. Proc Natl Acad Sci U S A 102:5588-5593.

Pellerin L, Bouzier-Sore AK, Aubert A, Serres S, Merle M, Costalat R, Magistretti PJ (2007) Activity-dependent regulation of energy metabolism by astrocytes: an update. Glia 55:1251-1262.

Porras OH, Loaiza A, Barros LF (2004) Glutamate mediates acute glucose transport inhibition in hippocampal neurons. J Neurosci 24:9669-9673.

Raichle ME, Mintun MA (2006) Brain work and brain imaging. Annu Rev Neurosci 29:449-476.

Rouach N, Koulakoff A, Abudara V, Willecke K, Giaume C (2008) Astroglial metabolic network sustain hippocampal synaptic transmission. Science 322:1551-1555.

Shimada M, Kawamoto S, Hirose Y, Nakanishi M, Watanabe H, Watanabe M (1994) Regional differences in glucose transport in the mouse hippocampus. Histochem J 26:207-212.

Sibson NR, Mason GF, Shen J, Cline GW, Herskovits AZ, Wall JE, Behar KL, Rothman DL, Shulman RG (2001) In vivo (13)C NMR measurement of neurotransmitter glutamate cycling, anaplerosis and TCA cycle flux in rat brain during [2-13C] glucose infusion. J Neurochem 76:975-989.

Sokoloff L (1977) Relation between physiological function and energy metabolism in the central nervous system. J Neurochem 29:13-26.

Somjen GG, Kager H, Wadman WJ (2008) Computer simulations of neuron-glia interactions mediated by ion flux. J Comput Neurosci 25: $349-365$.

Speizer L, Haugland R, Kutchai H (1985) Asymmetric transport of a fluorescent glucose analogue by human erythrocytes. Biochim Biophys Acta 815:75-84.

Voutsinos-Porche B, Bonvento G, Tanaka K, Steiner P, Welker E, Chatton JY, Magistretti PJ, Pellerin L (2003) Glial glutamate transporters mediate a functional metabolic crosstalk between neurons and astrocytes in the mouse developing cortex. Neuron 37:275-286.

Wyss MT, Weber B, Treyer V, Heer S, Pellerin L, Magistretti PJ, Buck A (2009) Stimulation-induced increases of astrocytic oxidative metabolism in rats and in humans investigated with 1-11C-acetate. J Cereb Blood Flow Metab 29:44-56.

Yamada K, Nakata M, Horimoto N, Saito M, Matsuoka H, Inagaki N (2000) Measurement of glucose uptake and intracellular calcium concentration in single, living pancreatic beta-cells. J Biol Chem 275:22278-22283. 